\title{
Survey Paper on Fine-Grained Facial Expression Recognition using Machine Learning
}

\author{
Gunjal Vaishnavi \\ JSPM's Bhivarabai Sawant \\ Institute of Technology and \\ Research, Wagholi, Pune
}

\author{
Gavane Shraddha \\ JSPM's Bhivarabai Sawant \\ Institute of Technology and \\ Research, Wagholi, Pune
}

\author{
Joshi Yogeshwari \\ JSPM's Bhivarabai Sawant \\ Institute of Technology and \\ Research, Wagholi, Pune
}

\begin{abstract}
A computer to monitor emotions that can assess fundamental speech of the human face. This research proposes a mood forecast based on emotions of the human face. The instrument to detect the human mood and to play an audio file with this effect that refers to human emotions. Next, the computer takes the human face as its input, so another move is taken. The face and eye are identified. This is done. The human face is then recognized by the technique of extraction of the attributes. In this way, a face picture feature recognizes the emotion of the individual. The lips, mouth and eyes and the eyebrow extract these signature marks. If the emotional face matches the emotional dataset exactly, the exact emotions of people can be defined to play the audio-file with the emotional details. Training on a limited number of faces would be recognized in different environmental circumstances. The proposed solution is quick, efficient and accurate. The machine plays an increasingly important part in the field of identification and detection.
\end{abstract}

\section{Keywords}

Face Detection, Feature Extraction, Face Emotion, Machine Learning.

\section{INTRODUCTION}

Face detection and authentication is one of human computer interactions' most important areas. The facial features are comparatively few and it is most important to research them. Face items have to be found and classified a challenging mission.

To find a human feeling in a person's face that can be one of the hardest things of your career. A face is the best way for a person to be seen and detected. No recognition algorithms can operate without face detection process. The rate of identity affects the stage of recognition. It's an intriguing work to detect and locate the invisible face of all these sounds in still images.

Detection of emotional mood is one of the subjects solving complex challenges in various fields. In addition to traditional problems in unchecked face pictures, such as varied poses, varying lighting, different facial gestures and various emotional sound levels. Mel's frequency portion is a database for all face and mood sensing devices, the most crucial item for comparison of face properties and sounds. Database construction facial features are calculated and stored in the database. The database is then used to measure the face and emotion by different algorithms.

Requests are often difficult for recognition of facial feelings, as face images may be affected by changes in the scene, which include variations in postures, postures or lighting. This approach is mostly designed to find human mood and then to play an audio file on the supporting face as an input. A facerecognition technique which compares the train face image with the original entrance image.

The proposed solution is quick, efficient and accurate. This system delivers accurate results compared to the current system. The machine plays an increasingly important part in the field of identification and detection. In comparison to traditional methods that produce useful results, this is very quick.

\section{LITERATURE SURVEY}

This work discusses many well documented and unique approaches for the extraction and emotional gradation of the facial expressions. Various algorithms are compared to performance parameters such as recognition precision, emotional quantity, experimental datasets, the description of facial expressions used [1].

This work identifies the face expressions of the face and classifies the thoughts as final judgement. The computer uses a basic facial positioning technique called 'Face Detection Viola

Jones.' The club uses a subset sorting technique to improve the accuracy of identifying and classifying processes with different features. The combined feature is eventually eligible and classified by the SVM, Random Forest and KNN classification technology[2]

This technique uses three phases of hair cascade recognition and features the removal by Active Form Model (ASM) and Adbots Classifiers technology of five emotional wrath, disgust, pleasure, neutrality and surprise[3].

This work uses an efficient approach to create a collection of face and emotional characteristics to describe the face and emotion. For the identification of the face from the input picture we use Viola-Jones face recognition methods and KNN classification techniques to analyses the face and emotion detection[4].

The purpose of this article is to show needs and applications for facial recognition. The interchange of facial expression between verbal and nonverbal means is not verbal but plays a major role. It represents the relationship or mental state of individuals [5].

In this suggested plan, the human face is interested in recognizing speech. There are several methods to identify the face image. This technique can be applied to the real process very easily. The computer shows short pictures from the webcam, senses the face and analyses the picture in such a way that there are little results [6]. 
In this work each frame is registered with an Avatar reference

face for a character, using the new SIFT flow technique. An iterative technique is used not only to super-resolved the EAI output for any video and avatar, but also to identify each video. In addition, the Local Binary Patch (LBP) method is applied to extract features from EAIs, using all Local Phase Quantization (LPQ) techniques[7].

In this analysis a system is built to recognize emotions that include facial recognition, characteristics extraction and facial expression classification. A skin detection system first of all assists the facial region in the face detection from a difficult setting. These functional points are initiated by tongue, mouth and eye recognition [8].

In this work, a new method is found to identify facial emotions. This proposal involves the use of the hair transformation technology and AdaBoost adaptive technique to identify face and component analysis (PCA) technique along with limited facial degree of recognition. Two methods have been validated for facial expression recognition. The first concerns the use of PCA and K-nearest (KNN) community technology, while the second encourages the use of the Negative Matrix Factorization (NMF) and KNN techniques [9].

\section{SYSTEM OVERVIEW}

The method suggested for identifying human emotions and then playing the human emotions audio file, with the result that news information can also be found through an API based upon user preferences. The first move of the computer is to take a snapshot of the human face. The face and eye are identified. This is done. The human face is subsequently known from the use of extraction methods for emotional processing. These techniques detect the emotion of man with the role of the face picture. The marking of the tongue, mouth and pupils and eyebrows shall indicate certain characteristic points. If the emotions-dependent data collection suits the input, human emotion can be detected for the emotional audio file exactly and on-line news information can also be collected using the API based on user preferences. Training on a limited number of features leads to an identification under different conditions in the field.

\section{ADVANTAGES PROPOSED SYSTEM:}

1. Face detection and cropping

2. Detection the mood of human using human face image features.

3. Play audio file using java based on human mood.

\section{METHODOLOGY}

Feature Extraction for Face Identification:

Color feature: As most of the color distribution information can be captured by the low-order moments, using only the first three moments: mean, variance and skewness, it is found that these moments give a good approximation and have been proven to be efficient and effective in representing the color distribution of images (Stricker and Oren go 1995).

Edge Detection: Most of the shape information of an image is enclosed in edges. So first we detect these edges in an image and by using these filters and then by enhancing those areas of image which contains edges, sharpness of the image will increase, and image will become clearer.

Texture feature: Describes the structure arrangement of surfaces and their relationship to the environment, such as fruit skin, clouds, trees, and fabric. The texture feature in our method is described by hierarchical wavelet packet descriptor (HWVP). A 170- D HWVP descriptor is utilized by setting the decomposition level to be 3 and the wavelet packet basis to be DB2.

\section{PROPOSED SYSTEM ARCHITECTURE}

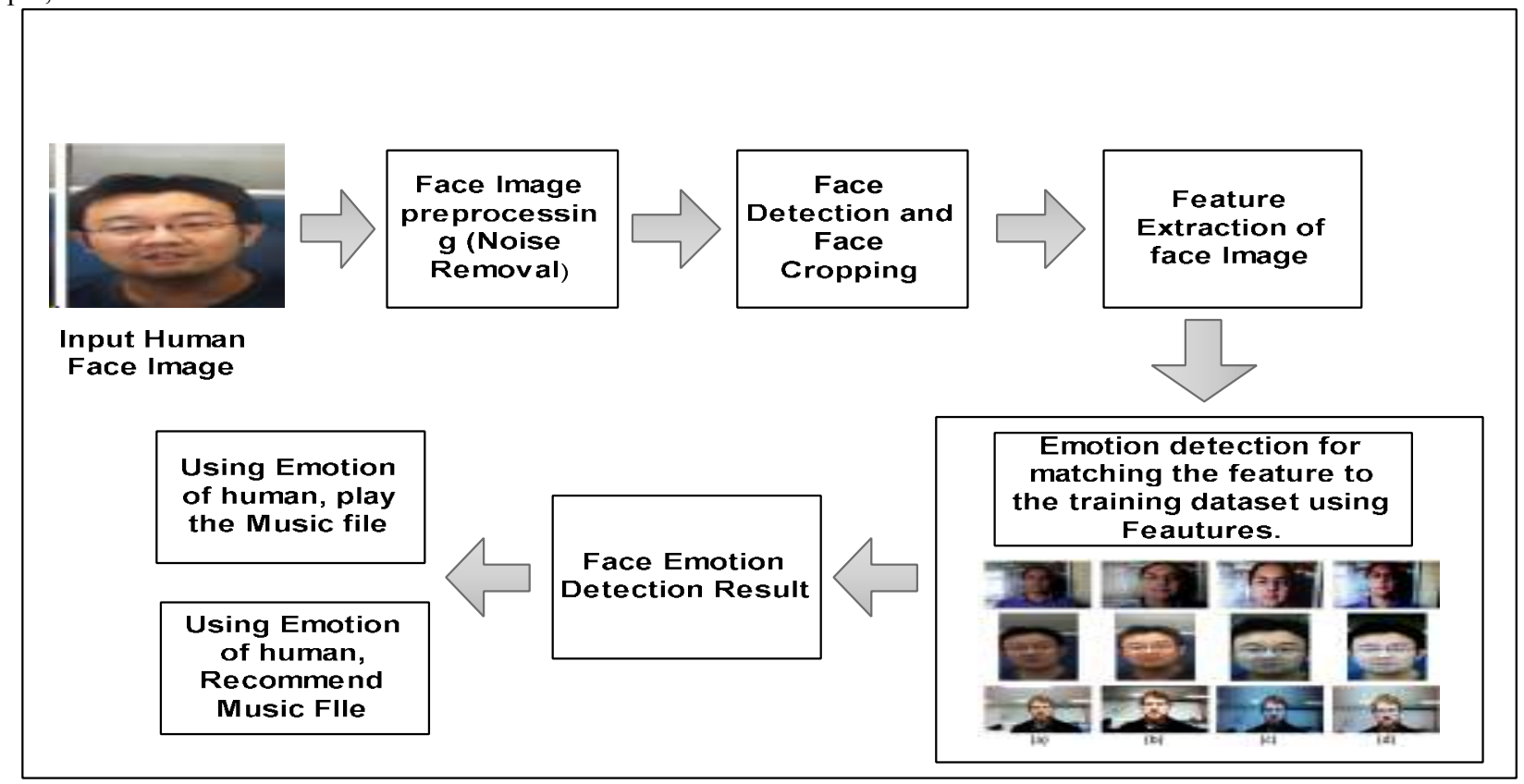

Fig1. Proposed System Architecture 


\section{MODULES:}

1. Input Image:

Here we will upload the Input face Image.

2. Image Pre-processing:

In this step we will applying the image pre-processing methods like gray scale conversion, image noise removal.

3. Image Feature Extraction:

In this step we will applying the image thresholding and edge detection methods to extract the image features from image.

4. Image Classification:

In this step we will applying the image classification methods to classify the face emotion.

5. Result:

In this step will show the final face emotion detections result.

\section{CONCLUSION}

Identifying a human emotion which relies on human face feels $\mathrm{n}$ has many applications in real life. The face of the pictures is hard to recognize. In this article a procedure for the detection of human emotion was suggested to adjust the algorithm using facial photography, to retrieve the characteristics and to fit the emotional training dataset for the human face. Once these findings have been used, we can also download news data from the API, the other user-dependent feature. The precision and time of recognition of large face datasets can be further extended. Our machine would also play a very important role in identifying human emotions.

\section{REFERENCES}

[1] Bharati Dixit, Arun Gaikwad, "Facial Features Based Emotion Recognition". ISSN (e): 2250-3021, ISSN (p): 2278-8719 Vol. 08, Issue 8 (August. 2018)

[2] J Jayalekshmi, Tessy Mathew, "Facial expression recognition and emotion classification system for sentiment analysis". 2017 International Conference.

[3] Suchitra, Suja P.Shikha Tripathi, "Real-time emotion recognition from facial images using Raspberry Pi II". 2016 3rd International Conference
[4] Dolly Reney, Neeta Tripathi, "An Efficient Method to Face and Emotion Detection". 2015 Fifth International Conference.

[5] Monika Dubey, Prof. Lokesh Singh, "Automatic Emotion Recognition Using Facial Expression: A Review". International Research Journal of Engineering and Technology (IRJET) Feb-2016.

[6] Anuradha Savadi Chandrakala V Patil, "Face Based Automatic Human Emotion Recognition". International Journal of Computer Science and Network Security, VOL.14 No.7, July 2014.

[7] Songfan Yang, Bir Bhanu, "Facial expression recognition using emotion avatar image". 2011 IEEE International Conference.

[8] LehLuoh, Chih-Chang Huang, Hsueh-Yen Liu, "Image processing based emotion recognition". 2010 International Conference.

[9] Jiequan Li, M. Oussalah, "Automatic face emotion recognition system". 2010 IEEE 9th International Conference.

[10] Megha Jadhav, Yogeshkumar Sharma and G M Bhandari. Forged Multinational Currency Identification and Detection System using Deep Learning Algorithm. International Journal of Computer Applications 177(44):36-40, March 2020.

[11] Megha Jadhav, Y. K. Sharma and G M Bhandari, "Currency Identification and Forged Banknote Detection using Deep Learning" 2019 International Conference on Innovative Trends and Advances in Engineering and Technology (ICITAET), Dec. 2019, pp 178-183.

[12] Jadhav M., Sharma Y., Bhandari G. (2021) Forged Multinational Currency Recognition System Using Convolutional Neural Network. In: Mahapatra R.P. Panigrahi B.K., Kaushik B.K., Roy S. (eds) Proceedings of 6 th International Conference on Recent Trends in Computing. Lecture Notes in Networks and Systems, vol 177. Springer, Singapore. 